\title{
EVALUATION OF THE ANTIBACTERIAL ACTIVITY OF CALCIUM ALGINATE BEADS MODIFIED WITH ETHANOLIC EXTRACT OF ADHATODA VASICA LEAF EXTRACT ON STAPHYLOCOCCUS AUREUS AND ESCHERICHIA COLI
}

\author{
DEEPA THOMAS ${ }^{1,3}$, LATHA M $\mathrm{S}^{2,3 *}$, KURIEN THOMAS $\mathrm{K}^{1}$
}

${ }^{1}$ Department of Chemistry, Bishop Moore College, Mavelikara, Kerala, India. ${ }^{2}$ Department of Chemistry, Sree Narayana College, Chengannur, Kerala, India. ${ }^{3}$ Department of Chemistry, Sree Narayana College, Kollam, Kerala, India. Email: lathams2014@gmail.com

Received: 27 October 2017, Revised and Accepted: 24 November 2017

\section{ABSTRACT}

Objective: The objective of the present study was to investigate the antibacterial activity of calcium alginate (Ca-ALG) loaded with ethanolic extract of Adhatoda vasica (A. vasica) leaves against Staphylococcus aureus (S. aureus) and Escherichia coli (E. coli).

Methods: Ca-ALG beads containing ethanolic extract of $A$. vasica leaves were developed by ionic gelation technique. The prepared Ca-ALG beads were characterized by Fourier-transform infrared (FT-IR) spectroscopy and scanning electron microscopy (SEM). The antibacterial effect of $A$. vasica leaf extract loaded Ca-ALG beads was examined against $S$. aureus and E. coli.

Results: FT-IR studies revealed the cross-linking of ALG and calcium ions. The spherical morphology of the beads was designated by SEM. The prepared beads were found to display distinctive growth inhibition against $S$. aureus and E. coli.

Conclusion: The antibacterial activity analysis indicated that the prepared beads have good activity against $S$. aureus and E. coli. The present study proposes a strategy to enhance antibacterial properties of ALG which are widely used in biomedical applications.

Keywords: Alginate, Adhatoda vasica, Ethanolic extract, Biomedical application, Antibacterial.

(C) 2018 The Authors. Published by Innovare Academic Sciences Pvt Ltd. This is an open access article under the CC BY license (http://creativecommons. org/licenses/by/4. 0/) DOI: http://dx.doi.org/10.22159/ajpcr.2018.v11i3.23367

\section{INTRODUCTION}

Natural polymers with antibacterial property receive considerable attention in the biomedical field. Usually, metal ions and metal nanoparticles are added to the system to improve its antibacterial activity. Cellular toxicity of such systems restricts its wide applications in the biomedical field [1]. The material utilized for biomedical application should be free of toxic chemicals and biocompatible in nature. The need of environmentally friendly and green antibacterial material intensifies the development of products of plant origin. The use of herbal extracts as therapeutic agents has numerous benefits such as biocompatibility, non-toxic, and eco-friendliness.

Adhatoda vasica (A. vasica) is a popular medicinal plant with wellknown antibacterial and antitussive activities [2,3]. It is an active ingredient of indigenous medicine for respiratory disorders. The leaf extract of $A$. vasica is used to cure chronic bronchial disorders, phthisis, cough, and asthma [4]. Earlier studies showed that it possesses excellent antibacterial activity [5]. The presence of quinazoline alkaloids such as vasicinone, vasicine, and deoxyvasicine is responsible for its medicinal value. Recent investigations reveal its bronchodilator activity [6].

Alginate (ALG) is a naturally occurring anionic polymer typically obtained from brown seaweed and is water soluble. It has the ability to gel in mild and aqueous conditions in the presence of divalent ions such as $\mathrm{Ca}^{2+}$ and $\mathrm{Zn}^{2+}$ and trivalent ions such as $\mathrm{Al}^{3+}$ and $\mathrm{Fe}^{3+}$ which leads to the formation of egg box junctions [7]. The biocompatible and non-toxic nature of ALG finds wide applications in the delivery of bioactive agents such as small chemical drugs and proteins, and cell transplantation [8-11]. ALG is well-known material in the wound management field due to its ability to maintain a moist environment and rich water content [12].

Among different metal cross-linked ALGs, calcium-ALG (Ca-ALG) was superior for wound healing applications. This is due to the unique hemostatic properties of the calcium ion and the ability of the gel to be used as a matrix for the aggregation of platelets and erythrocytes [13]. The porosity of ALG also allows the entrapment of bioactive agents and able to release it on the targeted site in a sustained and controlled manner. Usually, antibiotics such as minocycline, gentamycin, and ofloxacin are used for treating wound infections. The constant administration of these drugs may cause the resistance of the microorganisms to them. Antimicrobial dressings based on natural materials with therapeutic properties are the best solution to overcome this problem. The incorporation of medicinal plant extract into biocompatible polymer provides a unique opportunity for the development of novel therapeutic approaches toward biomedical application.

The aim of the present study is to prepare Ca-ALG beads loaded with ethanolic extract of $A$. vasica leaves by ionic gelation and examine its antibacterial activity against Staphylococcus aureus ( $S$. aureus) and Escherichia coli (E. coli). In this work, Ca-ALG beads loaded with ethanolic extract of $A$. vasica leaves were prepared by exploiting the hemostatic properties of ALG, and therapeutic activity of A. vasica leaves extract for wound healing and drug delivery applications.

\section{MATERIALS AND METHODS}

Materials

The materials used were sodium ALG (medium viscosity $\approx 500 \mathrm{cps}$, Sigma-Aldrich, London), calcium chloride dihydrate (Merck, Germany). All the other reagents were of analytical grade and used without further purification. A. vasica leaves were freshly collected from the college garden and identified with the help of Dr. Dinesh Raj, Department of Botany, Bishop Moore College, Mavelikara, Kerala.

\section{Preparation of extract}

Shade-dried leaves of $A$. vasica were coarsely powdered. About $250 \mathrm{~g}$ of this powder was extracted with $1000 \mathrm{ml}$ of $100 \%$ ethanol in an ultrasonicated 
water bath for 20 min and kept at room temperature for $24 \mathrm{~h}$. Then, the extract was filtered through Whatman No. 1 (pore size $25 \mu \mathrm{m}$ ) filter paper. The residue was subjected to re-extraction for 3 times using fresh ethanol, and the combined filtrates were evaporated to dryness under reduced pressure $(20-50 \mathrm{k} \mathrm{Pa})$ at $40^{\circ} \mathrm{C}$ using a Buchi rotary evaporator. The dark green residue was finally dried in vacuum (yield $20 \mathrm{~g}$ ) [14]

\section{Preparation of $\boldsymbol{A}$. vasica leaf extract loaded Ca-ALG beads}

Sodium ALG was dissolved in $10 \mathrm{ml}$ deionized water (3\%, w/v). About $200 \mathrm{mg} \mathrm{A}$. vasica leaf extract is added to this solution and stirred for $30 \mathrm{~min}$ to obtain a homogenous solution. The homogenized solution was dropped into $1 \%$ calcium chloride aqueous solution using a $25-\mathrm{G}$ needle from $5 \mathrm{~cm}$ distance at room temperature. The droplets were kept in solution for $20 \mathrm{~h}$. Then, the $A$. vasica leaf extract loaded Ca-ALG beads was separated from the solution, washed several times with distilled water and dried.

\section{Scanning electron microscopy (SEM)}

The surface morphology of the A. vasica leaf extract loaded Ca-ALG bead was imaged using a JEOL JSM-6390LA Analytical Scanning Electron Microscope.

\section{Fourier-transform infrared (FT-IR) analysis}

FT-IR studies of sodium ALG and Ca-ALG were carried out using Shimadzu FT-IR model 1801 between 400 and $4000 \mathrm{~cm}^{-1}$ wavelength range to ensure the cross-linking between ALG and $\mathrm{Ca}^{2+}$ ions.

\section{Evaluation of antibacterial activity}

Antibacterial activity of $A$. vasica leaf extract loaded Ca-ALG beads was evaluated against $S$. aureus and $E$. coli. Petri plates containing $20 \mathrm{ml}$ MullerHinton agar medium were seeded with bacterial culture of $E$. coli and $S$. aureus (growth of culture adjusted according to McFards standard, $0.5 \%$ ). Wells of approximately $10 \mathrm{~mm}$ were bored using a well cutter, and samples of desired concentrations were added. All the plates were incubated for 24 $\mathrm{h}$ at $37^{\circ} \mathrm{C}$. The antibacterial activity was tested by measuring the diameter of the zone of inhibition formed around the well (NCCLS, 1993).

\section{RESULTS AND DISCUSSION}

Plant extracts are viewed as a promising material for biomedical applications since it possesses excellent antiviral, antifungal, antiinflammatory, antioxidant, and antibacterial activity due to the presence of biologically active ingredients. Among them, A. vasica is well known for its medicinal properties and is easily obtainable and economical. In the present study, we prepared the ethanolic extract of $A$. vasica leaves loaded in Ca-ALG with the aim to develop a biomaterial with remarkable antibacterial activity. Previous reports from various research groups justify the choice of ethanol as solvent in our extraction $[15,16]$

ALG is an easily available and inexpensive natural polymer with wellestablished biocompatibility and non-toxicity. A. vasica leaf extract loaded Ca-ALG beads was prepared by ionotropic gelation technique. Ionotropic gelation technique is the most common and simple method for the preparation of ALG-based beads. Here, all the preparation steps were performed in mild and aqueous conditions and free from toxic organic solvents. These factors assure the biocompatibility of the prepared ALG beads.

The SEM image of the prepared $A$. vasica leaf extract loaded Ca-ALG bead is shown in Fig. 1. It confirms the spherical shape of the obtained bead. The ionotropic gelation method gave beads with a spherical shape. The divalent calcium ions form a chelated structure with the polyguluronate unit of ALG molecule, leads to the formation of spherical beads [17].

The FT-IR spectra of Na-ALG and Ca-ALG were taken and are shown in Fig. 2 . The band at $3445 \mathrm{~cm}^{-1}$ in the spectra of the sodium ALG can be assigned to the stretching vibrations of the $\mathrm{O}-\mathrm{H}$ bond. The peaks around 1649 and $1460 \mathrm{~cm}^{-1}$ were assigned to asymmetric and symmetric stretching vibrations of carboxylate ions. In Ca-ALG beads, the peak corresponding to the $\mathrm{O}-\mathrm{H}$ bond becomes narrow. This is caused by the formation of chelating structure between hydroxyl and carboxylate groups of ALG with calcium ion. As a result of this chelating structure formation, the asymmetric stretching vibration of ALG is also shifted from $1649 \mathrm{~cm}^{-1}$ to $1625 \mathrm{~cm}^{-1}$ [18].

The result of the antibacterial activity study of $A$. vasica leaf extract loaded Ca-ALG bead against $S$. aureus and E. coli is shown in Fig. 3. The result suggests that the prepared $A$. vasica leaf extract loaded Ca-ALG bead displayed antibacterial activity against $S$. aureus (inhibition zone of $A$. vasica leaf extract loaded Ca-ALG bead - $1.5 \pm 0.10 \mathrm{~cm}$, negative control dimethyl sulfoxide (DMSO) - $0.00 \pm 0.00$, and positive control streptomycin - 2.85 $\pm 0.05 \mathrm{~cm}$ ) and $E$. coli (inhibition zone of $A$. vasica leaf extract loaded CaALG bead $-12 \pm 1.9 \mathrm{~cm}$, negative control DMSO - $0.00 \pm 0.00$, and positive control streptomycin $-2.74 \pm 0.18 \mathrm{~cm}$ ). In previous studies, Patel and Venkatakrishna-Bhatt reported the antimicrobial activity of $A$. vasica [19].

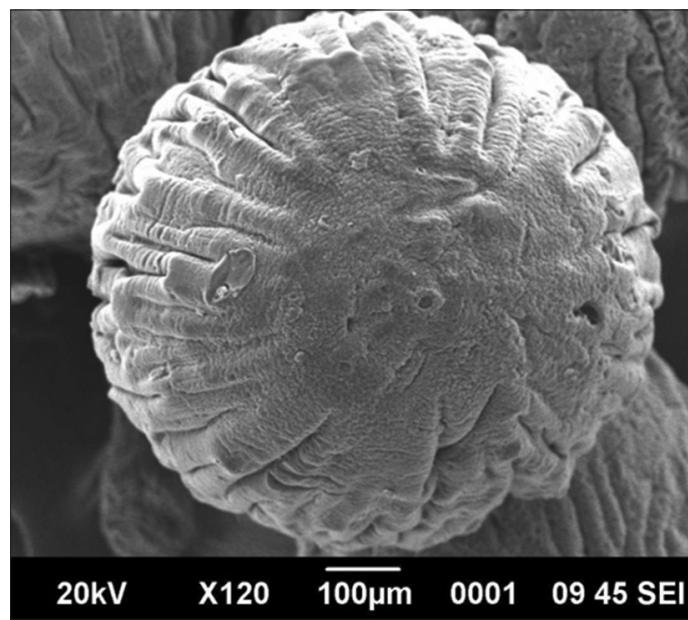

Fig. 1: Scanning electron microscopic image of Adhatoda vasica leaf extract loaded calcium alginate bead

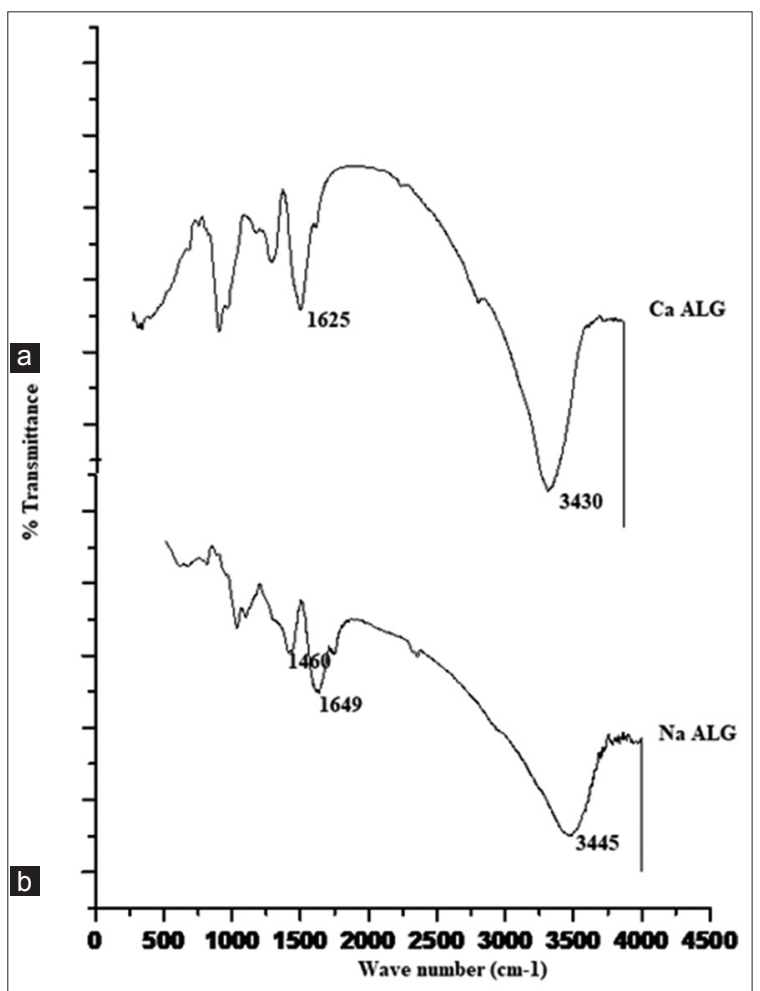

Fig. 2: Fourier-transform infrared spectra of (a) calcium-alginate (b) Na-alginate 

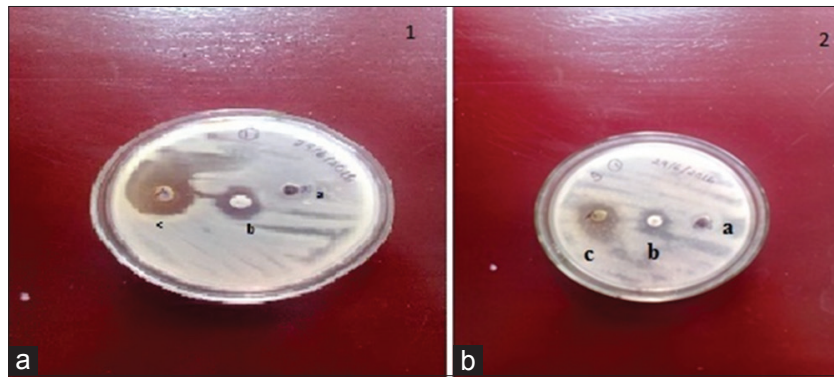

Fig. 3: Antibacterial activity of Adhatoda vasica leaf extract loaded calcium-alginate (Ca-ALG) bead against Staphylococcus aureus (plate 1) and Escherichia coli (plate 2). In both the plates (a) negative control dimethyl sulfoxide, (b) A. vasica leaf extract loaded Ca-ALG bead, and (c) positive control streptomycin

The resultant material can find excellent application in the field of wound healing. A. vasica leaf extract can act as a barrier against pathogens and prevent the growth of microorganisms. In addition to the antibacterial activity, A. vasica leaf extract also showed well wound healing activity [20]. In earlier studies, Bhargava et al. proved the potential of alcoholic extract as a wound healing agent in buffaloes [21]. They reported the ability of A. vasica for improving tensile strength, extensibility, and absorption in the wound repair tissue. They also reported that $A$. vasica can enhance the amount of elastin, collagen, hydroxyproline, hexosamine, and zinc. The porous nature of Ca-ALG allows the gaseous exchange in and out of the wound surface. All these properties make the prepared sample suitable for wound healing applications.

\section{CONCLUSION}

In the present study, a rapid and simple methodology was proposed to prepare $A$. vasica leaf extract loaded CA-ALG beads by ionotropic gelation technique. The spherical morphology of the prepared beads was proved by SEM analysis. The successful cross-linking between the carboxyl group of ALG and calcium ions was confirmed by FT-IR analysis. The antibacterial activity analysis indicated that the prepared beads have good activity against $S$. aureus and $E$. coli. The preliminary study proposes that the prepared material has suitable properties for wound healing application. The study offers a new strategy for developing wound healing material.

\section{ACKNOWLEDGMENTS}

Deepa Thomas acknowledges the University Grants Commission (FIP/12 ${ }^{\text {th }}$ plan/KLKE002TF05) for the financial support. The authors are thankful to Sophisticated Test and Instrumentation Centre, Cochin, for providing FT-IR and SEM analysis facilities.

\section{AUTHORS CONTRIBUTION}

Deepa Thomas and M.S. Latha conceived and designed the experiments.

Deepa Thomas prepared the samples and performed the experiments.

Deepa Thomas, M.S. Latha and K.Kurien Thomas worked together on the development of the ideas presented in this paper, and contributed to the data analysis and manuscript writing.

\section{CONFLICT OF INTEREST}

The authors declare no conflict of interest.

\section{REFERENCES}

1. Samberg ME, Loboa EG, Oldenburg SJ, Monteiro-Riviere NA. Silver nanoparticles do not influence stem cell differentiation but cause minimal toxicity. Nanomedicine 2012;7:1197-209.

2. Mathew AS, Patel KN, Shah BK. Investigation on antifeedant and anthelmentic potential of Adhatoda vasica nees. Indian J Nat Prod Resour 1998; 14:11-6.

3. Dhuley JN. Antitussive effect of Adhatoda vasica extract on mechanical or chemical stimulation-induced coughing in animals. J Ethnopharmacol 1999;67:361-5.

4. Amin AH, Mehta DR. A bronchodilator alkaloid (vasicinone) from Adhatoda vasica nees. Nature 1959;184 Suppl 17:1317.

5. Singh B, Sharma RV. Anti-inflammatory and antimicrobial properties of pyrroloquinazoline alkaloids from Adhatoda vasica nees. Phytomedicine 2013;20:441-5.

6. Kumar KPS, Bhowmik D, Chiranjib, Tiwari P, Kharel R. Indian traditional herbs Adhatoda vasica and its medicinal application. J Chem Pharm Res 2010;2:240-5.

7. Hamed S, Ayob FA, Alfatama M, Doolaanea AA. Enhancement of the immediate release of paracetamol from alginate beads. Int J Appl Pharm 2017;9:47-51.

8. Lopes MA, Abrahim-Vieira B, Oliveira C, Fonte P, Souza AM, Lira T, et al. Probing insulin bioactivity in oral nanoparticles produced by ultrasonication-assisted emulsification/internal gelation. Int $\mathrm{J}$ Nanomedicine 2015;10:5865-80.

9. Marie Arockianathan P, Sekar S, Sankar S, Kumaran B, Sastry TP. Evaluation of biocomposite films containing alginate and sago starch impregnated with silver nano particles. Carbohydr Polym 2012;90:717-24

10. Madhavi C, Kumarababu P, Maruthi Y, Parandhama A, Reddy OS, Rao KC, et al. Sodium alginate-locust bean gum IPN hydrogel beads for the controlled delivery of nimesulide-anti-inflammatory drug. Int $\mathbf{J}$ Pharm Pharm Sci 2017;9:245-52

11. Reddy KV, Nagabhushanam MV. Process and parameters affecting drug release performance of prepared cross-linked alginate hydrogel beads for ezetimibe. Int J Pharm Pharm Sci 2017;9:254-62.

12. Paul W, Sharma CP. Chitosan and alginate wound dressing: A short review. Trends Biomater Artif Organs 2004;18:18-23.

13. Boateng JS, Matthews KH, Stevens HNE, Eccleston GM. Wound healing dressings and drug delivery systems: A review. J Pharm Sci 2008;97:2893-923

14. Singh A, Kumar S, Reddy TJ, Rameshkumar KB, Kumar B. Screening of tricyclic quinazoline alkaloids in the alkaloidal fraction of adhatoda beddomei and Adhatoda vasica leaves by high-performance liquid chromatography/electrospray ionization quadrupole time-offlight tandem mass spectrometry. Rapid Commun Mass Spectrom 2015;29:485-96.

15. Pandit K, Langfield RD. Antibacterial activity of some Italian medicinal plant. J Ethano Pharm 2004;82:135-42.

16. Karthikeyan A, Shanthi V, Nagasathaya A. Preliminary phytochemical and antibacterial screening of crude extract of the leaf of Adhatoda vasica (L). Int J Green Pharm 2009;3:78-80.

17. Suganya VS, Asheeba ST. Microencapsulation of astaxanthin using ionotropic gelation method isolated from three crab varieties. Int J Curr Pharm Res 2015;7:96-9.

18. Daemi H, Barikani M. Synthesis and characterization of calcium alginate nanoparticles, sodium homopolymannuronate salt and its calcium nanoparticles. Sci Iran 2012;19:2023-8.

19. Patel VK, Venkatakrishna-Bhatt H. In vitro study of antimicrobial activity of Adhatoda vasika linn. (leaf extract) on gingival inflammation - a preliminary report. Indian J Med Sci 1984;38:70-2.

20. Gangwar AK, Ghosh AK. Medicinal uses and pharmacological activity of Adhatoda vasica. Int J Herb Med 2014;2:88-91.

21. Bhargava MK, Singh H, Kumar A. Evaluation of Adhatoda vasica as a wound healing agent in buffaloes - Clinical, mechanical and biochemical studies. Indian Vet J 1988;65:33-8. 